\title{
Variation in CT perfusion protocol has implications on defining irreversibly damaged ischemic brain parenchyma
}

\author{
Jai Jai Shiva Shankar ${ }^{1}$ (D)
}

Received: 12 June 2021 / Revised: 29 June 2021 / Accepted: 4 July 2021 / Published online: 30 July 2021

(C) European Society of Radiology 2021

\section{Key Points}

- Computed tomographic perfusion (CTP) is increasingly being used in the characterization of brain ischemia.

- Variations in post-processing protocols continue to be a challenge, resulting in a slight variation of CTP results.

- We need to adopt a universal acquisition protocol to help optimize output of CTP.

Computed tomographic perfusion (CTP) is increasingly being used in the characterization of brain ischemia [1]. Most commonly, these are used for acute ischemic stroke for the characterization of infarct core and ischemic penumbra. More recently, CT perfusion is also being used for the characterization of ischemic injury in critically ill patients - such as those suspected to have brain death in ICU [2, 3], comatose cardiac arrest patients [4], and those with severe traumatic brain injury $[5,6]$.

In their European Radiology article, Peerlings et al report the variations in post-processing protocols continue to be a challenge, resulting in a slight variation of CTP results [7]. The significance of variation depends on whether we look for penumbra (potentially reversible ischemia) or core (irreversible ischemic damage). To define the irreversibly damaged ischemic core, the generally acceptable threshold for cerebral blood flow (CBF) has been $15 \mathrm{~mL} / \mathrm{min} / 100 \mathrm{~g}$ of brain tissue and that for cerebral blood volume (CBV) of $2 \mathrm{~mL} / 100$ $\mathrm{g}$ of brain tissue $[8,9]$. When comparing $\mathrm{CBF}$ and $\mathrm{CBV}, \mathrm{CBV}$ is more likely to predict the irreversibly damaged ischemic core; i.e., if brain region is dead on CBV maps, it is unlikely to recover on follow-up irrespective of treatment $[1,8]$. On the other hand, relative $\mathrm{CBF}$ is more sensitive in predicting the irreversibly damaged ischemic core [9].

This comment refers to the article available at https://doi.org/10.1007/ s00330-021-08067-6

Jai Jai Shiva Shankar

shivajai1@gmail.com

1 Rady Faculty of Health Sciences, Radiology Department, University of Manitoba, GA216-820 Sherbrook Street, Winnipeg, MB R3A 1R9, Canada
The perfusion parameters are calculated based on the time density curve (TDC) that depicts the rate of change of density of contrast during the first pass of the contrast bolus. The TDC is a function of the injection of the contrast bolus, heart rate, and cardiac output. The faster the contrast injection rate, the tighter the contrast bolus, which results in a sharper, narrower, and higher TDC. The faster the heart rate, the sharper and narrower the TDC. The corollary is also true for patients with slow heart rate and low cardiac output. Controlling patient physiological parameters such as heart rate and cardiac output is usually not done in the clinical setting where CTP is used. But the injection rate of contrast agent could be changed and should be standardized to compare CTP results across patients as well as across centers. Bolus arrival time and TDC width are good surrogate markers for the patient's physiology. A patient with a slower heart rate and a lower cardiac output usually has a longer bolus arrival time and a wider TDC.

This brings to attention another important CTP acquisition parameter, time of acquisition. If the total time of acquisition is not long enough, the whole cardiac cycle may not be covered during CTP acquisition, resulting in the truncation of the TDC. The shorter time of acquisition is largely because of excessive concerns of radiation dose. Recent guidelines have suggested a longer time of acquisition of 70-90 s to avoid any possibilities of truncation of TDC [10]. CBV is calculated as the area under the curve of TDC. CBF is calculated based on the ascending segment of TDC. Truncation of TDC negatively affects the calculation of $\mathrm{CBV}$ resulting in under-estimation of $\mathrm{CBV}$, thereby over calling the volume of irreversibly damaged ischemic core. This has minimal to no effect in the calculation of CBF. Truncation of TDC is not uncommon and can be seen in up to $30 \%$ of cases [9]. This has resulted in transition from $\mathrm{CBV}$ to $\mathrm{CBF}$ as the preferred parameter to predict the 
irreversibly damaged ischemic core [9]. A relative $\mathrm{CBF}$ is better than absolute $\mathrm{CBV}$ in depicting the irreversibly damaged ischemic core. A threshold of absolute CBF of $<15 \mathrm{~mL} / 100 \mathrm{~g} / \mathrm{min}$ correlates with that of irreversibly damaged ischemic core [9].

Effects of truncation of TDC are also significant when CTP is used to predict devastating brain injury in patients other than those with ischemic stroke, e.g., those with severe traumatic head injury, comatose cardiac arrest, and suspected brain death [2-6]. Truncation artifacts can be controlled by increasing the time of acquisition of CTP, which results only in a miniscule percentage increase in the associated patient radiation dose.

As the use of CTP for ischemic stroke is increasing and the scope of CTP is expanding, a better understanding of the different factors that could influence the results of CTP is needed. With a better understanding, we need to adopt a universal acquisition protocol to help optimize output of CTP.

Funding The author states that this work has not received any funding.

\section{Declarations}

Guarantor The scientific guarantor of this publication is Dr Jai Shankar.

Conflict of interest The author of this manuscript declares no relationships with any companies whose products or services may be related to the subject matter of the article.

Statistics and biometry No complex statistical methods were necessary for this paper.

Informed consent Written informed consent was not required for this study because it is an editorial.

Ethical approval Institutional Review Board approval was not required because this is an editorial.

\section{Methodology}

- $\quad$ Editorial comment

\section{References}

1. Shankar JJS, Langlands G, Doucette S, Phillips S (2016) CT perfusion in acute stroke predicts final infarct volume- inter-observer study. Can J Neurol Sci 43:93-97

2. Shankar J, Vandorpe R (2013) CT Perfusion for confirmation of brain death. AJNR Am J Neuroradiol 34:1175-1179

3. MacDonald D, Stewart-Perrin B, Shankar JJS (2018) The role of neuroimaging in the determination of brain death. J Neuroimaging 28:374-379

4. Shankar JJS, Stewart-Perrin B, Quraishi A-U-R, Bata I, Vandorpe $\mathrm{R}$ (2018) Computed tomography perfusion aids in the prognostication of comatose postcardiac arrest patients. Am J Cardiol 121:874 878

5. Shankar JJS, Green R, Virani K, Wong H, Eddy K, Vandorpe R (2020) Admission perfusion CT for classifying early in-hospital mortality of patients with severe traumatic brain injury: a pilot study. AJR Am J Roentgenol 214(4):872-876

6. Alcock S, Batoo D, Ande SR et al (2021) Early diagnosis of mortality using admission $\mathrm{CT}$ perfusion in severe traumatic brain injury patients (ACT-TBI): protocol for a prospective cohort study. BMJ Open 11:e047305. https://doi.org/10.1136/bmjopen-2020-047305

7. Peerlings D, Bennink E, Dankbaar JW, Vethuis BK, de Jong HWAM, DUtch acute STroke (DUST) study investigators (2021) Variation in arterial input function in a large multicenter computed tomography perfusion study. Eur Radiol https://doi.org/10.1007/ s00330-021-08067-6.

8. Wintermark M, Flanders AE, Velthuis B et al (2006) Perfusion-CT assessment of infarct core and penumbra: receiver operating characteristic curve analysis in 130 patients suspected of acute hemispheric stroke. Stroke 37:979-985

9. Campbell BCV, Christensen S, Levi CR et al (2011) Cerebral blood flow is the optimal CT perfusion parameter for assessing infarct core. Stroke 42:3435-3440

10. Wintermark M, Albers GW, Broderick JP et al (2013) Acute stroke imaging research roadmap II. Stroke 44:2628-2639

Publisher's note Springer Nature remains neutral with regard to jurisdictional claims in published maps and institutional affiliations. 